ORIGINAL

\title{
Ocorrência de lesões podais em bovinos de corte criados em lotação contínua no estado do Pará
}

\author{
Occurrence of claw diseases in beef cattle raised on continued grazing \\ in Pará state
}

\footnotetext{
Rinaldo Batista Viana ${ }^{*}$, Bruno Moura Monteiro², Waldjânio de Oliveira Melo², Daniel Rocha de Oliveira ${ }^{3}$, Luciara Celi Chaves Daher ${ }^{1}$, José Dantas Ribeiro Filho ${ }^{4}$

1 Instituto da Saúde e Produção Animal, Universidade Federal Rural da Amazônia (UFRA), Belém, PA, Brasil

2 Universidade Federal Rural da Amazônia (UFRA), Paragominas, PA, Brasil

${ }^{3}$ Agência Estadual de Defesa Agropecuária do Estado do Pará (ADEPARA), Santarém, PA, Brasil

${ }^{4}$ Universidade Federal de Viçosa (UFV), Viçosa, MG, Brasil
}

\section{Resumo}

As injúrias sofridas pelos dígitos dos bovinos são altamente dolorosas e, quando não tratadas, levam a sérios problemas sanitários, afetando diretamente a produtividade e o bem-estar dos animais. A prevalência dessas enfermidades já é bem estabelecida em vacas leiteiras, todavia, no gado de corte em varias regiões do país pouco se sabe sobre sua ocorrência. Deste modo, objetivou-se realizar um estudo sobre a ocorrência de doenças podais em bovinos de corte no estado do Pará. Foram utilizados nesse estudo os cascos de 1318 bovinos (1108 fêmeas e 210 machos) advindos de diversos municípios e mesorregiões do estado do Pará, obtidos em um abatedouro localizado em Belém. Após o abate, cada unha foi individualmente examinada para diagnóstico e eventual classificação das doenças. A análise estatística dos dados foi conduzida com base no teste de Quiquadrado $\left(\mathrm{x}^{2}\right)$, com nível de significância de $5 \%$, a fim de quantificar e qualificar as lesões podais. Observou-se que 26,25\% (346/1318) dos bovinos avaliados apresentavam pelo menos uma lesão em uma das regiões do casco, sendo que nos bovinos criados em lotação contínua as enfermidades dos dígitos mais prevalentes foram a hiperplasia interdigital, doença da linha branca (fissura e abscesso de linha branca) e erosão de talão. A incidência de lesões podais foi maior em fêmeas.

Palavras-chave: Bovino. Claudicação. Pododermatite. Casco. Unha. 


\section{Abstract}

Injuries to bovine hooves are highly painful and lead to serious health problems when left untreated, directly affecting animal productivity and welfare. The prevalence of these diseases is already well established in dairy cattle, but little is known about their occurrence in beef cattle from several regions of Brazil. The purpose of this study was to research the occurrence of foot diseases in beef cattle in Pará state, Brazil. The hooves of 1,318 cattle (1,108 female and 210 male) from several municipalities and mesoregions of Pará state were obtained in a slaughterhouse located in Belém. After the slaughter, each claw was individually examined for diagnosis and eventual classification of the disease. Statistical analysis of the data was performed based on the Chi-square test $\left(x^{2}\right)$, with significance level of $5 \%$, in order to quantify and qualify the foot diseases. It was observed that $26.25 \%$ (346/1318) of the evaluated cattle had at least one lesion in one of the hull regions. In cattle kept under continuous grazing, the most prevalent diseases were interdigital hyperplasia, white line disease (white line fissure and abscess) and heel erosion. In addition, the incidence of foot injuries was higher in females.

Keywords: Bovine. Lameness. Pododermatitis. Hoof. Claw.

\section{Introdução}

As doenças dos dígitos dos bovinos estão entre as enfermidades mais prevalentes em granjas leiteiras. Embora existam muitos inquéritos epidemiológicos descritos com altas prevalências, da ordem de $14,13 \%$ (Martis et al., 2002) a 22,25\% (Silveira et al., 2009), a maioria destes estudos tem sido realizada em vacas leiteiras. Tomasella et al. (2014) evidenciaram que 8,5 \% das vacas lactantes estudadas apresentavam algum tipo de lesão podal, demonstrando claudicação. Entre os sinais clínicos apresentados nas doenças podais, a claudicação é o mais presente, visto que $90 \%$ dos bovinos leiteiros com claudicação têm desordens localizadas nos cascos (Murray et al., 1996).

Apesar de existirem alguns inquéritos sobre a prevalência das doenças podais em bovinos de corte, nenhum desses foi realizado na Amazônia Oriental, sobretudo em rebanhos de bovinos de corte criados no estado do Pará. Ademais, das duas únicas pesquisas feitas, apenas uma realizou estudos sobre as enfermidades podais em bovinos de corte criados a pasto, e considerou apenas a região sudeste paraense (Silveira et al., 2018); o outro determinou a prevalência de afecções podais em vacas da bacia leiteira no município de Rondon do Pará (Silveira et al., 2009).

Em uma pesquisa realizada já há duas décadas e meia, em bovinos de corte no estado do Rio de Janeiro, Ribeiro et al. (1992) citaram à época uma baixa prevalência $(0,88 \%)$ de pododermatites em bovinos de corte. Possivelmente, essa não seja mais a realidade das fazendas de corte brasileiras, visto que enfermidades infectocontagiosas como a dermatite digital também se encontram disseminadas nas fazendas de bovinos de corte.

Dado os sérios problemas causados pelas doenças podais, como emagrecimento e perda de peso, descarte precoce dos animais e comprometimento da eficiência reprodutiva (Morris et al., 2011), além do aumento dos níveis de estresse, visto que a claudicação é considerada um sinal de más condições de bem-estar dos animais, há a necessidade do conhecimento dos aspectos da epidemiologia da doença, sobretudo nos grandes rebanhos, a fim de se implementar medidas eficazes de controle e profilaxia.

Nesse sentido, o presente trabalho objetivou determinar a ocorrência das enfermidades podais em bovinos de corte criados em lotação contínua no estado do Pará, verificando quais enfermidades apresentaram maiores ocorrências e qual a influência do sexo na ocorrência destas lesões.

\section{Materiais e métodos}

0 estudo foi realizado em um abatedouro localizado no município de Belém, utilizando-se informações de 1318 bovinos mestiços de corte (1108 fêmeas e 210 machos) criados em lotação contínua em fazendas localizadas no estado do Pará (Tabela 1).

Foram recolhidos e identificados os cascos (5272) dos animais na linha de abate, sendo estes avaliados individualmente em áreas, consideradas como unidade amostral: unhas (10544) e espaços 
interdigitais (5272). Deste modo, avaliaram-se 15816 áreas dos cascos examinadas (unhas + espaços interdigitais), das quais nove tiveram suas informações perdidas durante a colheita dos dados, totalizando, assim, 15807 resultados coligidos e analisados.

Tabela 1 - Número de bovinos incluídos no estudo de acordo com a mesorregião e município de origem

\begin{tabular}{|c|c|c|}
\hline Mesorregião & Município & $\begin{array}{l}\text { Número de animais } \\
\text { incluídos no estudo }\end{array}$ \\
\hline \multirow[t]{6}{*}{ Ilha de Marajó } & Chaves & 4 \\
\hline & Ponta de Pedras & 12 \\
\hline & Irituia & 84 \\
\hline & Garrafão do Norte & 7 \\
\hline & Bonito & 9 \\
\hline & Tomé-Açu & 21 \\
\hline \multirow[t]{14}{*}{ Nordeste Paraense } & Santa Luzia do Pará & 10 \\
\hline & Concórdia do Pará & 25 \\
\hline & Ulianópolis & 33 \\
\hline & Tailândia & 20 \\
\hline & Peixe Boi & 5 \\
\hline & Ipixuna do Pará & 17 \\
\hline & Jacundá & 75 \\
\hline & Breu Branco & 20 \\
\hline & São Geraldo do Araguaia & 186 \\
\hline & Rio Maria & 62 \\
\hline & Água Azul do Norte & 55 \\
\hline & Paragominas & 63 \\
\hline & Goianésia do Pará & 101 \\
\hline & Xinguara & 31 \\
\hline \multirow[t]{8}{*}{ Sudeste Paraense } & Bom Jesus do Tocantins & 33 \\
\hline & $\begin{array}{l}\text { São Domingos do } \\
\text { Araguaia }\end{array}$ & 44 \\
\hline & Rondon do Pará & 71 \\
\hline & Novo Repartimento & 9 \\
\hline & Marabá & 102 \\
\hline & Bannach & 42 \\
\hline & Sapucaia & 11 \\
\hline & Itupiranga & 10 \\
\hline Sudoeste Paraense & Pacajá & 156 \\
\hline Total de bovinos & & 1318 \\
\hline
\end{tabular}

As doenças podais foram classificadas segundo Borges et al. (2017): unha assimétrica (UA); parede dorsal côncava (PC); unha em saca-rolha (SR); dermatite digital (DD) ou dermatite papilomatosa; dermatite interdigital (DI) ou superficial (frieira); sola dupla (SD); erosão de talão (ET) (podridão do casco); fissura da parede axial (FPA); fissura horizontal da parede (FHP); fissura vertical da parede (FVP); hiperplasia interdigital (HI); flegmão interdigital (FI) (panarício); unha em tesoura (UT) ; hemorragia difusa da sola (HDS); hemorragia circunscrita da sola (HCS); edema de coroa e/ou bulbo (ECB); úlcera de sola (US); úlcera de bulbo (UB); úlcera de pinça (UP); necrose de pinça (NP); sola fina (SF); fissura de linha branca (FLB); abscesso de linha branca (ALB); pododermatite séptica (broca, podridão do casco); pododermatite do paradígito (necrose da sobreunha, arranquio da sobreunha); tungíase (bicho-de-pé); e miíase (bicheira).

\section{Análise estatística}

Os dados de ocorrência de lesões foram apresentados como frequência $(\%, n / n)$. As ocorrências de lesões foram comparadas de acordo com as variáveis classificatórias: sexo, área do casco (unhas e espaço interdigital), classificação da doença, plano transversal (membros anteriores e posteriores), plano mediano (membros direito e esquerdo), unhas (lateral e medial), assim como as interações entre as mesmas. A análise estatística dos dados foi conduzida com base no teste de Quiquadrado $\left(\mathrm{x}^{2}\right)$, considerando o nível de significância de $5 \%$, a fim de verificar associação significativa entre sexo e cada uma das variáveis estudadas.

\section{Resultados e discussão}

Observou-se que 26,25\% (346/1318) dos bovinos incluídos na pesquisa apresentavam pelo menos uma lesão em uma das áreas do casco. 0 número elevado de animais acometidos por lesões podais pode estar associado a uma combinação de fatores, como clima úmido e tipo de solo da região estudada, uma vez que elevada umidade é fator predisponente na gênese das afecções podais.(Silva et al.,2001). 
Dentre as áreas dos cascos examinadas (unhas e espaços interdigitais), verificou-se que 3,43\% (542/15807) delas apresentavam enfermidades podais (Tabela 2). Das 15807 áreas dos cascos examinadas, pode-se observar que $3,07 \%$ (485/15807) de todas as lesões podais foram diagnosticados nas vacas, enquanto apenas $0,36 \%$ (57/15807) foram diagnosticadas nos machos (Tabela 2). Portanto, $89,48 \%$ do total de unhas e espaços interdigitais acometidos por algum tipo de doença podal foram diagnosticados nas fêmeas (Figura 1) e somente 10,52\% nos machos. Considerando-se o porcentual em cada categoria (fêmeas x machos) (Tabela 2), 3,65\% das unhas e espaços interdigitais das vacas apresentavam doenças podais (485/13287), enquanto nos machos esse valor foi igual a 2,26\% (57/2520). Evidenciou-se, portanto, maior ocorrência de doenças dos dígitos em função do sexo $(\mathrm{p}=0,0004)$.

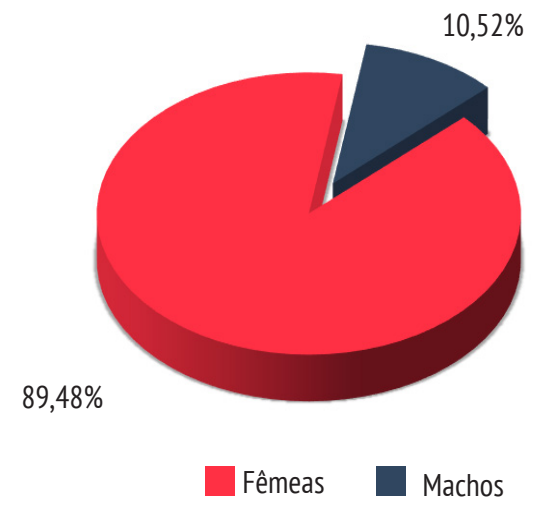

Figura 1 - Porcentagem de lesões podais em bovinos de corte criados em sistema de lotação contínua no estado do Pará, de acordo com o sexo dos animais.

Essa influência do sexo na ocorrência de doenças dos dígitos nos bovinos estudados pode, possivelmente, ser atribuída ao fato de que os animais abatidos em alguns frigoríficos no estado do Pará são vacas de descarte (Melo et al., 2015). Leão et al. (2009) descreveram que a incidência de dermatite digital tem relação com a idade e citaram maior frequência em animais entre 3 e 6 anos, o que justificaria, inclusive, o descarte dessas vacas.

Considerando-se o plano transversal do corpo dos animais, observou-se que as áreas dos cascos dos membros anteriores foram mais acometidas por doenças podais $(54,43 \%)$ em relação aos posteriores $(45,57 \%)$ (Figura 2), diferentemente dos resultados encontrados por Silveira et al. (2018) em bovinos de corte criados na mesorregião sudeste paraense. Todavia, ao se considerar o plano mediano do corpo dos animais, as lesões apresentaram iguais distribuições entre os membros direitos $(48,52 \%)$ e esquerdos $(51,48 \%)$ $(p=0,4834)$ (Figura 3), divergindo dos resultados de Tomasella et al. (2014).

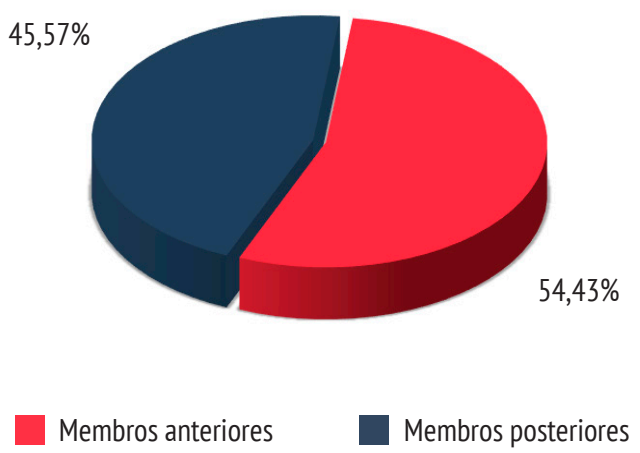

Figura 2 - Porcentagem de lesões podais em bovinos de corte criados em sistema de lotação contínua no estado do Pará, de acordo com plano transversal do corpo dos animais (membros anteriores $\mathrm{x}$ posteriores).

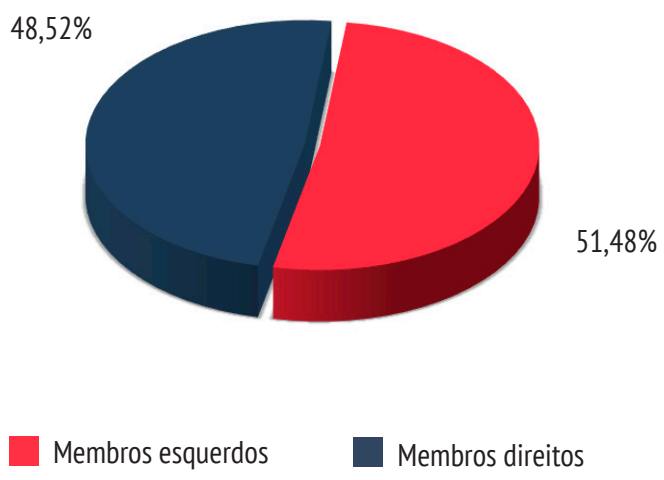

Figura 3 - Porcentagem de lesões podais em bovinos de corte criados em sistema de lotação contínua no estado do Pará, de acordo com plano mediano do corpo dos animais (membros esquerdos $\mathrm{x}$ direitos). 
Tabela 2 - Porcentagem de lesões podais nas unhas e espaços interdigitais em bovinos de corte criados em sistema de lotação contínua no estado do Pará, de acordo com o sexo e o membro acometido

\begin{tabular}{lcccc}
\hline \multicolumn{5}{c}{ Lesões podais nas unhas e espaços interdigitais } \\
\hline \multirow{3}{*}{ Variável } & $\begin{array}{c}\text { Unhas e espaços } \\
\text { interdigitais sem lesões }\end{array}$ & $\begin{array}{c}\text { Unhas e espaços } \\
\text { interdigitais com lesões }\end{array}$ \\
\cline { 2 - 5 } & $\mathbf{\%}$ & $\mathbf{n}$ & $\%$ & $\mathbf{n}$ \\
\hline Fêmeas & $80,99(96,35)$ & 12802 & $3,07(3,65)$ & 485 \\
Machos & $15,58(97,74)$ & 2463 & $0,36(2,26)$ & 57 \\
Total & 96,57 & 15265 & 3,43 & 542 \\
Anteriores & $48,14(96,27)$ & 7609 & $1,87(3,73)$ & 295 \\
Posteriores & $48,43(96,87)$ & 7656 & $1,56(3,13)$ & 247 \\
Total & 96,57 & 15265 & 3,43 & 542 \\
Direitos & $48,34(96,67)$ & 7641 & $1,66(3,33)$ & 263 \\
Esquerdos & $48,23(96,47)$ & 7624 & $1,77(3,53)$ & 279 \\
Total & 96,57 & 15265 & 3,43 & 542 \\
\hline
\end{tabular}

Nota: Qui-quadrado. Áreas do casco nos machos x áreas do casco fêmeas, $p=0,0004$. Áreas do casco nos membros anteriores $x$ áreas do casco nos membros posteriores, $p=0,0360$. Áreas do casco nos membros direitos $x$ áreas do casco nos membros esquerdos, $p=0,4834$. Porcentagens calculadas a partir do total de unhas e espaços interdigitais examinados $(n=15807)$. Valores entre parênteses indicam porcentagem de lesões calculadas, considerando-se 0 total de lesões em cada variável individualmente (fêmeas $=13287$; machos $=2520$; anteriores $=7904$; posteriores $=7903$; direitos $=7904$; esquerdos $=7903$ ).

Nas fêmeas, o maior porcentual de lesões se deu nas unhas e espaços interdigitais dos membros anteriores $(4,03 \% ; 268 / 6646)$ frente aos posteriores $(3,27 \% ; 217 / 6641)$, ou seja, $55,26 \%$ das lesões estavam localizadas nos membros anteriores e $44,74 \%$ nos membros posteriores ( $\mathrm{p}=0,0187)$. Já nos machos não houve diferença $(p=0,6967)$ na prevalência de lesões em função do membro acometido (anteriores: 2,15 \%; 27/1259; posteriores: 2,38\%; 30/1262). Todavia, nota-se uma tendência, uma vez que $52,63 \%$ das lesões foram diagnosticadas nos membros posteriores (fêmeas + machos), frente aos $47,37 \%$ das lesões nos membros anteriores.

Atribui-se a maior ocorrência de lesões nos membros anteriores ao fato de que no presente estudo as fêmeas foram mais acometidas por lesões nos cascos do que os machos, e estas apresentaram maior porcentual de lesões nos membros anteriores, contrapondo-se ao descrito por Silveira et al. (2018), que descreveram maior prevalência de lesões nos membros posteriores. Possivelmente, diversos fatores concorrem para a distribuição do peso corpóreo de vacas, entre eles o estágio gestacional em que o útero gravídico poderia distribuir maior peso nos membros pélvicos, visto que o peso do útero com o feto e os líquidos placentários varia de 40 a $80 \mathrm{~kg}$ no final da gestação (Ferreira, 2010). As vacas não gestantes incluídas no presente estudo apresentariam, portanto, distribuição do peso corpóreo de forma mais homogênea. Tal resultado incomum pode também estar associado ao sistema de criação adotado, onde predominam pastos sujos, irregulares, com restos de troncos de árvores, capim seco, etc, e aos maiores frame sizes (estrutura corporal) atribuídos a fêmeas selecionadas para sistemas de produção de corte, os quais estabelecem uma conformação corporal das vacas mais equilibrada, com maior simetria dianteira-traseira. Além disso, há pouca discrepância entre as medidas corpóreas de fêmeas bovinas de corte, tais como largura peitoral x largura do íleo e, ainda, alta correlação entre estas medidas, para quaisquer classes de estrutura corpórea (média, grande ou extrema) (Mota el al., 2015).

As unhas foram mais acometidas do que os espaços interdigitais (Tabela 3; Figura 4), com 3,83\% (404/10544) e 2,62\% (138/5263) de ocorrência de lesões, respectivamente, culminando em 74,54\% das lesões nas unhas $(41,51 \%$ nas anteriores e $33,03 \%$ nas posteriores) e $25,46 \%$ nos espaços interdigitais $(\mathrm{p}<0,0001)$. Entre as unhas, as mediais apresentaram maior incidência de lesões $(4,08 \%$; $215 / 5270)$ frente às laterais $(3,58 \% ; 189 / 5274)$, em que $39,67 \%$ das lesões ocorreram nas unhas mediais e $34,87 \%$ nas laterais $(p=0,0002)$.

A pressão suportada pela unha atinge valores altos durante a locomoção sobre uma superfície plana e dura, e a agressão resultante sofrida pelo estojo córneo pode ser uma das causas das desordens de cascos (van der Tol et al., 2003), o que pode explicar o fato de as unhas terem sido mais lesionadas do que os espaços interdigitais nos animais estudados. 
Percebe-se que as unhas mediais, tanto no dígito esquerdo quanto no direito, apresentaram maiores porcentuais de lesões do que as laterais nos membros anteriores. Já nos membros posteriores, a maior ocorrência de lesões se deu nas unhas laterais $(\mathrm{p}=0,0018)$. Esse comportamento ocorre devido à diferenciação do estresse de sustentação de peso entre as unhas, de forma que o casco medial suporta maior peso nos membros torácicos, enquanto o casco lateral sustenta mais peso nos membros pélvicos, já que apresenta maior área de pressão, o que interfere positivamente na ocorrência de lesões nesses dígitos (van der Tol et al., 2003; Peek e Divers, 2018).

Tabela 3 - Porcentagem de lesões podais em bovinos de corte criados em sistema de lotação contínua no estado do Pará, de acordo a região do casco afetada

\begin{tabular}{lcccc}
\hline \multicolumn{5}{c}{ Porcentagem e número de lesões podais } \\
\hline \multirow{2}{*}{ Área do casco } & Sem lesões & Com lesões \\
\cline { 2 - 5 } & $\%$ & $n$ & $\%$ & $\mathbf{n}$ \\
\hline UADL $(n=1317)$ & $8,00(95,98)$ & 1264 & $0,33(4,02)$ & 53 \\
UADM $(n=1321)$ & $7,99(95,61)$ & 1263 & $0,37(4,39)$ & 58 \\
UAEL $(n=1320)$ & $8,04(96,29)$ & 1271 & $0,31(3,71)$ & 49 \\
UAEM $(n=1315)$ & $7,91(95,06)$ & 1250 & $0,41(4,94)$ & 65 \\
UPDL $(n=1317)$ & $8,06(96,74)$ & 1274 & $0,27(3,26)$ & 43 \\
UPDM $(n=1317)$ & $8,08(96,96)$ & 1277 & $0,25(3,04)$ & 40 \\
UPEL $(n=1321)$ & $8,00(95,69)$ & 1264 & $0,36(4,31)$ & 57 \\
UPEM $(n=1316)$ & $8,08(97,04)$ & 1277 & $0,25(2,96)$ & 39 \\
EIAD $(n=1316)$ & $8,10(97,26)$ & 1280 & $0,23(2,74)$ & 36 \\
EIAE $(n=1315)$ & $8,10(97,41)$ & 1281 & $0,22(2,59)$ & 34 \\
EIPD $(n=1316)$ & $8,12(97,49)$ & 1283 & $0,21(2,51)$ & 33 \\
EIPE $(n=1316)$ & $8,10(97,34)$ & 1281 & $0,22(2,66)$ & 35 \\
\hline Total $(n=15807)$ & 96,57 & 15265 & 3,43 & 542 \\
\hline
\end{tabular}

Nota: Qui-quadrado: $p=0,0018$. Porcentagens calculadas a partir do total de unhas e espaços interdigitais examinados. Valores entre parênteses indicam porcentagem de lesões calculadas, considerando-se o total de lesões em cada área do casco individualmente. UADL - unha anterior direita lateral; UADM - unha anterior direita medial ; UAEL - unha anterior esquerda lateral; UAEM - unha anterior esquerda medial; UPDL - unha posterior direita lateral; UPDM - unha posterior direita medial; UPEL - unha posterior esquerda lateral; UPEM - unha posterior esquerda medial; EIAD - espaço interdigital anterior direito; EIAE - espaço interdigital anterior esquerdo; EIPD - espaço interdigital posterior direito; EIPE - espaço interdigital posterior esquerdo.

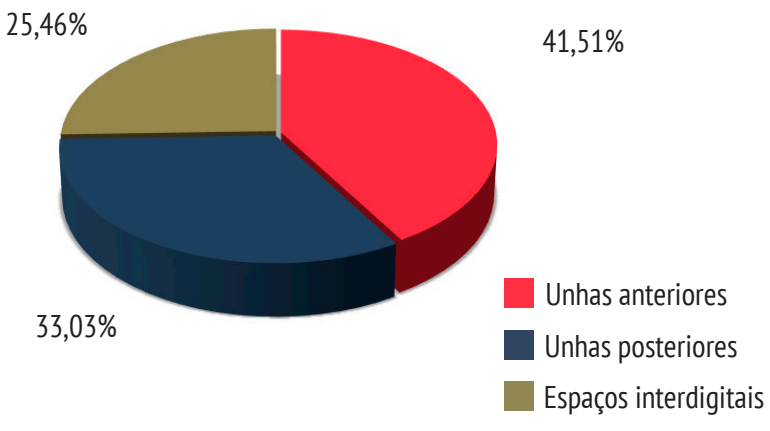

Figura 4 -Porcentagem de lesões podais em bovinos de corte criados em sistema de lotação contínua no estado do Pará, de acordo com a área do casco (unhas anteriores $\mathrm{x}$ unhas posteriores $x$ espaços interdigitais.

Nos espaços interdigitais, a HI foi a doença mais prevalente $(2,24 \% ; 118 / 5263)$. Já nas unhas, tanto lateral quanto medial, as doenças mais prevalentes foram ET $(1,16 \%$ para a unha lateral e 1,00\% para unha medial; $61 / 5270$ e 53/5274, respectivamente) e UT, com $0,82 \%(43 / 5270)$ do total de lesões em ambas as unhas $(\mathrm{p}<0,0001)$ (Tabela 4$)$.

Considerando apenas o total de lesões em cada categoria animal, observou-se ocorrência de HI igual a $0,81 \%(108 / 13287)$ nas fêmeas e $0,40 \%$ $(10 / 2520)$ nos machos, enquanto a ET apresentou ocorrências iguais a 0,78\% (104/13287) e 0,40 $(10 / 2520)$ em fêmeas e machos, respectivamente.

Verificou-se que a doença podal de maior ocorrência nos machos foi a da linha branca (FLB e ALB); já nas fêmeas, a HI foi a doença mais prevalente ( $p=0,0020)$. Silveira et al. (2009) obtiveram resultado semelhante ao avaliarem vacas da bacia leiteira de Rondon do Pará, onde as lesões de HI também foram as mais frequentes e se caracterizaram pela proliferação da pele do espaço interdigital, presente, na maioria das vezes, em mais de um membro, com intensidade entre leve (sem claudicação) e grave (com ulceração e claudicação). 0 efeito exercido pela forragem seca sobre o espaço interdigital provavelmente contribuiu para a ocorrência da doença, especialmente em animais criados em pastagens em solos íngremes e irregulares, onde o capim seco causa traumatismo crônico do espaço interdigital posterior devido à abertura das unhas ao impulsionar o corpo durante a subida (Alvim et al., 2005). 
Tabela 4 - Distribuição das doenças dos dígitos de bovinos de corte criados em sistema de lotação contínua no estado do Pará, de acordo com o sexo, o membro acometido e a área do casco acometida

\begin{tabular}{|c|c|c|c|c|c|c|c|c|c|c|c|c|c|c|}
\hline \multicolumn{15}{|c|}{ Distribuição das doenças dos dígitos } \\
\hline \multirow{3}{*}{ Doença } & \multicolumn{4}{|c|}{ Sexo } & \multicolumn{4}{|c|}{ Membros } & \multicolumn{6}{|c|}{ Área do casco } \\
\hline & \multicolumn{2}{|c|}{ Fêmea } & \multicolumn{2}{|c|}{ Macho } & \multicolumn{2}{|c|}{ Anteriores } & \multicolumn{2}{|c|}{ Posteriores } & \multicolumn{2}{|c|}{ Espaço interdigital } & \multicolumn{2}{|c|}{ Unha lateral } & \multicolumn{2}{|c|}{ Unha medial } \\
\hline & $\%$ & $\mathrm{n}$ & $\%$ & $\mathrm{n}$ & $\%$ & $\mathrm{n}$ & $\%$ & $\mathrm{n}$ & $\%$ & $\mathrm{n}$ & $\%$ & $\mathrm{n}$ & $\%$ & $\mathrm{n}$ \\
\hline$A D$ & 0,01 & 2 & 0,00 & 0 & 0,00 & 0 & 0,01 & 2 & 0,00 & 0 & 0,01 & 1 & 0,01 & 1 \\
\hline $\mathrm{DD}$ & 0,06 & 9 & 0,01 & 1 & 0,03 & 5 & 0,03 & 5 & 0,04 & 7 & 0,01 & 1 & 0,01 & 2 \\
\hline DI & 0,08 & 12 & 0,01 & 1 & 0,02 & 3 & 0,06 & 10 & 0,08 & 13 & 0,00 & 0 & 0,00 & 0 \\
\hline ET & 0,66 & 104 & 0,06 & 10 & 0,53 & 84 & 0,19 & 30 & 0,00 & 0 & 0,39 & 61 & 0,34 & 53 \\
\hline FLB e ALB & 0,21 & 33 & 0,09 & 14 & 0,16 & 25 & 0,14 & 22 & 0,00 & 0 & 0,14 & 22 & 0,16 & 26 \\
\hline FHP e FVP & 0,23 & 36 & 0,06 & 9 & 0,16 & 26 & 0,12 & 19 & 0,00 & 0 & 0,17 & 27 & 0,11 & 18 \\
\hline $\mathrm{Fl}$ & 0,01 & 1 & 0,00 & 0 & 0,01 & 1 & 0,00 & 0 & 0,00 & 0 & 0,00 & 0 & 0,00 & 0 \\
\hline HDS e HCS & 0,19 & 30 & 0,02 & 3 & 0,06 & 9 & 0,15 & 24 & 0,00 & 0 & 0,13 & 20 & 0,08 & 13 \\
\hline $\mathrm{HI}$ & 0,68 & 108 & 0,06 & 10 & 0,40 & 63 & 0,35 & 55 & 0,75 & 118 & 0,00 & 0 & 0,00 & 0 \\
\hline PS & 0,04 & 6 & 0,01 & 1 & 0,01 & 2 & 0,03 & 5 & 0,00 & 0 & 0,02 & 3 & 0,03 & 4 \\
\hline SD & 0,09 & 15 & 0,01 & 2 & 0,03 & 5 & 0,08 & 12 & 0,00 & 0 & 0,06 & 9 & 0,05 & 8 \\
\hline UP & 0,12 & 19 & 0,02 & 3 & 0,11 & 18 & 0,03 & 4 & 0,00 & 0 & 0,08 & 13 & 0,06 & 9 \\
\hline US & 0,01 & 2 & 0,01 & 1 & 0,01 & 1 & 0,01 & 2 & 0,00 & 0 & 0,01 & 2 & 0,01 & 1 \\
\hline UA & 0,11 & 17 & 0,00 & 0 & 0,03 & 4 & 0,08 & 13 & 0,00 & 0 & 0,06 & 9 & 0,05 & 8 \\
\hline$S R$ & 0,04 & 7 & 0,00 & 0 & 0,02 & 3 & 0,03 & 4 & 0,00 & 0 & 0,03 & 4 & 0,02 & 3 \\
\hline UT & 0,53 & 84 & 0,01 & 2 & 0,29 & 46 & 0,25 & 40 & 0,00 & 0 & 0,27 & 43 & 0,27 & 43 \\
\hline Total & 3,07 & 485 & 0,37 & 57 & 1,87 & 295 & 1,56 & 247 & 0,87 & 138 & 1,38 & 215 & 1,2 & 189 \\
\hline
\end{tabular}

Nota: Qui-quadrado: $p=0,0020$ (machos $x$ fêmeas), $p<0,0001$ (membros anteriores $x$ posteriores), $p<0,0001$ (área do casco). Ausência do dígito (AD); Dermatite digital (DD); Dermatite interdigital (DI); Erosão de talão (ET); Fissura (FLB) e Abscesso de linha branca (ALB); Fissura horizontal (FHP) e vertical da parede (FVP); Flegmão interdigital (FI); Hemorragia difusa (HDS) e Circunscrita da sola (HCS); Hiperplasia interdigital (HI); Pododermatite séptica (PS); Sola dupla (SD); Úlcera de pinça (UP); Úlcera de sola (US); Unha assimétrica (UA); Unha em saca-rolha (SR); Unha em tesoura (UT).

\section{Conclusão}

Nos bovinos criados em lotação contínua no estado do Pará, as enfermidades dos dígitos mais prevalente são a hiperplasia interdigital, doença da linha branca (fissura e abscesso de linha branca) e erosão de talão. As fêmeas são mais acometidas do que os machos.

\section{Referências}

Alvim NC, Bento MAF, Cunha Filho LFC, Souto JHB. Hiperplasia interdigital em bovinos. Rev Cient Eletr Med Vet. 2005;1(5):1-6.
Borges JRJ, Câmara ACL, Moscardini ARC, Rodrigues CA, Pitombo CA, Graça FAS, et al. Doenças dos dígitos dos bovinos: nomenclatura padronizada para o Brasil. Revista CFMV. 2017;23(73):45-52.

Ferreira AM. Gestação em bovinos. Reprodução da Fêmea Bovina: Fisiologia apli-cada e problemas mais comuns. Juiz de Fora: UFV; 2010. p. 345-94.

Leão MA, Silva LAF, Jayme VS, Silva LM, Moura MI, Barbosa VT. Aspectos epidemi-ológicos da dermatite digital bovina em duas propriedades produtoras de leite do estado de Goiás, Brasil. Cienc Anim Bras. 2009;10(4);1135-47. 
Martins CF, Sarti E, Busato I, Pires PP, Fiori CH, Moreira $C$, et al. Prevalência e classificação das afecções podais em vacas lactantes na bacia leiteira de Campo Grande (Capital) e municípios arredores - MS. Ensaios e Ci. 2002;6(2):113-37.

Melo WO, Santos EA, Abud LJ, Coelho GJ, Santos SC, Almeida LRR, et al. Impacto econômico da ocorrência de lesões em carcaças de bovinos abatidos no sudeste do Pará. Acta Vet Bras. 2015;9(3):243-50.

Morris MJ, Kaneko K, Walker SL, Jones DN, Routly JE, Smith RF, et al. Influence of lameness on follicular growth, ovulation, reproductive hormone concentrations and estrus behavior in dairy cows. Theriogenology. 2011;76(4):658-68.

Mota LFM, Mariz TMA, Ribeiro JS, Silva MEF, Lima Jr DM. Divergência morfométrica em bovinos nelore em crescimento classificados para diferentes classes de frame size. Rev Caatinga. 2015;28(2):117-25.

Murray RD, Downham DY, Clarkson MJ, Faull WB, Hughes JW, Manson FJ, et al. Epidemiology of lameness in dairy cattle: description and analysis of foot lesions. Vet Rec. 1996;138(24):586-91.

Peek SF, Divers TJ. Rebhun's Diseases of Dairy Cattle. 3 ed. St. Louis: Elsevier; 2018. 704 p.

Ribeiro PN, Borges JRJ, Ronconi MA, Marsico Filho F, Pitombo CA. Incidência de afecções podais em bovinos de corte abatidos no Estado do Rio de Janeiro. Arq EMVUFBA. 1992;15:28-33.
Silva LAF, Silva LM, Romani AF, Rabelo RE, Fioravanti MCS, Souza TM, Silva CA. Características clínicas e epidemiológicos das enfermidades podais em vacas lactantes do município de Orizona/GO. Cienc Anim Bras. 2001;2(2):119-26.

Silveira JAS, Albernaz TT, Oliveira CMC, Duarte MD, Barbosa JD. Afecções podais em vacas da bacia leiteira de Rondon do Pará. Pesq Vet Bras. 2009;29(11):905-9.

Silveira JAS, Silva NS, Albernaz TT, Bomjardim HA, Reis ASB, Oliveira CMC, et al. Epidemiological and clinical study of foot diseases in beef cattle extensive management in southeastern Pará, Brazil. Pesq Vet Bras. 2018;38(3):367-73.

Tomasella TE, Negri Filho LC, Affonso MZ, Barca Jr F, Silva LC, Okano W. Preva-lência e classificações de lesões podais em bovinos leiteiros na região de Belo Ho-rizonteMG. Rev Bras Hig Sanid Anim. 2014;8(1):115-27.

van der Tol PPJ, Metz JHM, Noordhuizen-Stassen EN, Back W, Braam CR, Weijs WA. The vertical ground reaction force and the pressure distribution on the claws of dairy cows while walking on a flat substrate. J Dairy Sci. 2003;86(9); 2875-83. 